\title{
PROKLA-Redaktion
}

\section{Editorial: Ausnahmezustand: Barbarei oder Sozialismus?}

Die gegenwärtige Situation ist schwer zu fassen. Dieser "Ausnahmezustand“ bedarf selbst einer kritischen Analyse. Dabei ist mit „Ausnahmezustand“ nicht einfach eine Herrschaftspraxis jenseits rechtsstaatlicher Prinzipien gemeint, die es zunehmend auch gibt, sondern eine zeitdiagnostische Feststellung: die Welt scheint aus den Fugen. Diese Feststellung war der Ausgangspunkt und die Motivation des vorliegenden Heftes. Im PROKLA-Schwerpunkt „Ausnahmezustand - Barbarei oder Sozialismus?" sollten die Dynamiken und Zusammenhänge dieser nicht leicht einzuordnenden Ausnahmesituation genauer analysiert werden. Welche Entwicklungslinien zeichnen sich derzeit $\mathrm{ab}$ ? Von welchen innergesellschaftlichen und internationalen Kräftekonstellationen werden sie getragen? Welche Widersprüche und Konflikte liegen ihnen zugrunde? Und stehen die vielen beunruhigenden Ereignisse, die auf den ersten Blick nichts miteinander zu tun zu haben scheinen, nicht vielmehr doch in einem (welt-)politischen Zusammenhang, den eine Analyse herausstellen müsste? - so lauteten die Leitfragen unseres ambitionierten Projekts, dem wir nur bedingt gerecht werden konnten. Vieles ist in der Schwebe und Entwicklungen kaum abzusehen. Zusammenhänge nicht nur zu behaupten, sondern auch aufzuzeigen und zu analysieren, bedarf nicht nur der Zeit, sondern auch der Sachkenntnisse von meist recht unterschiedlichen politischen
Feldern, die sonst meist getrennt voneinander betrachtet und analysiert werden. Resultat ist, dass wir als Redaktion der PROKLA, neben den in diesem Heft versammelten Aufsätzen, nach vielen Jahren wieder einen gemeinsamen, längeren Text als Redaktionskollektiv verfasst haben. $\mathrm{Ob}$ wir der selbst gesteckten Aufgabe gerecht geworden sind, soll die kritische Leserschaft entscheiden.

Die Debatte über Krise, Krisenverläufe und -folgen vollzieht sich in den letzten Jahren oft im Rahmen von Vergleichen - etwa mit der Krise von 1929. Ingo Schmidt diskutiert in seinem Beitrag aus vergleichender Perspektive die beiden Krisen und folgenden Stagnationsphasen und stellt heraus, dass in den Bearbeitungsformen der Krise ab 1929 vor allem die Existenz der Sowjetunion als Machtfaktor einen entscheidenden Unterschied bedeutete. Sein Hauptargument ist, dass die antisystemischen Bewegungen, die in den 1930er Jahren existierten und nach dem Zweiten Weltkrieg an Boden gewannen, das Kapital drängten, sich von der imperialistischen Expansion und interimperialistischer Rivalität ab- und einer Durchdringung der heimischen Märkte zuzuwenden. Das machte ein starkes Wirtschaftswachstum und einen sozialen Kompromiss möglich, der zugleich hohe Gewinne zuließ. Demgegenüber sei die Ursache für die aktuelle Stagnation, dass diese Möglichkeit ausgeschöpft ist. Ohne 
systemische Herausforderungen hat das Kapital keinen Grund, seine Akkumulationsstrategien grundlegend in eine Richtung zu verändern, die zur Überwindung der Stagnation beitragen könnten. Stattdessen stützt es seine Profite aufKosten der subalternen Klassen, auch wenn dies die Stagnation verlängert und zu schärferen gesellschaftlichen Spaltungen führt.

Lukas Oberndorfer zeichnet nach, wie der Wettbewerbsetatismus in Frankreich autoritär durchgesetzt wurde und welche Rolle der dort nach den Terroranschlägen vom November 2015 ausgerufene Ausnahmezustand dabei spielte: Grundrechte wurden aufgehoben, um die Deregulierung des Arbeitsmarktes voranzutreiben. Im Rahmen eines hegemonietheoretischen Ansatzes zeigt er, dass diese Entwicklung nur im Zusammenhang des europäischen Staatsapparate-Ensembles zu verstehen ist. Er kritisiert vor diesem Hintergrund den wissenschaftlichen Mainstream, der aufgrund seines eurozentrischen und liberalen Forschungsdesigns den Autoritarismus des Ausnahmezustands nur als äußere Bedrohung begreift - also nicht als eine Tendenz, welche - im Anschluss an Nicos Poulantzas' Überlegungen zu autoritärem Etatismus, Normalform des kapitalistischen Staats und Ausnahmestaat - dem modernen bürgerlichen Staat und der Demokratie selbst innewohnt.

Franziska Schutzbach widmet sich in ihrem Text dem Antigenderismus, wie er gegenwärtig nicht nur auf Demonstrationen von sogenannten „Lebensschützern“, sondern auch von Vertreter_innen der AfD bis hinein ins bürgerliche Feuilleton artikuliert wird. Die zentralen Argumentationsmuster sind stets die gleichen: das Bedrohungsszenario der Auflösung geschlechtlicher Identität und der traditionellen Familie durch den Feminismus, das Bild einer totalitären gesellschaftlichen
Gleichmacherei sowie die Diskreditierung von Gender Studies als „unwissenschaftlich“. Antigenderismus, so Schutzbach, funktioniert dabei gegenwärtig als „gemeinsamer und verbindendender Nenner für verschiedene rechtskonservative und neoliberale Kräfte“. Das Pochen auf natürliche Ordnungen schafft Allianzen zwischen neoliberalen und neokonservativen Bestrebungen und macht diese anschlussfähig an Rassismus, Nationalismus und an ein Programm staatlicher Disziplinierung. Die gegenwärtige Konjunktur antigenderistischer Programmatiken und der Propagierung der Familie kontextualisiert sie schließlich in der vermehrten (popkulturellen) Sichtbarkeit des Feminismus einerseits und in Erfahrungen der Prekarisierung am Arbeitsplatz, in den familialen Lebensweisen und vergeschlechtlichten Subjektivitäten andererseits.

Für Jason Moore ist die Thematisierung der Naturverhältnisse zentraler Bestandteil von Kapitalismuskritik. Dem derzeit populären Begriff des Anthropozäns setzt er den des Kapitalozäns entgegen. Nicht „der Mensch“, eine begriffslose Abstraktion, sondern das Kapital sei der treibende und maßgebliche Faktor dafür, dass sich die Naturverhältnisse seit dem 15. Jahrhundert grundlegend umwälzten. Umgekehrt sei die Krise des Kapitalverhältnisses nicht ohne die Naturverhältnisse zu erfassen. „Die kapitalistischen Ursprünge zu verstehen“, so Moore in seinem Aufsatz, ,ist eine tückische Arbeit."Das Schulbuchbild, das wir von der Industriellen Revolution kennen, muss „durch jene der globalen Transformationen von menschlichen und nicht-menschlichen Naturen ergänzt werden, die sich in den letzten fünf Jahrhunderten durch die neuen Sicht- und Organisationsweisen der unbezahlten Arbeit von Menschen und der sonstigen Natur herausgebildet haben. " All das ist 
insofern besonders bedenkenswert, als eine geologische Kommission im August diesen Jahres empfohlen hat, „Anthropozän“zur offiziellen Bezeichnung des Zeitalters zu machen, in das die Menschheit spätestens seit Mitte des 20. Jahrhunderts eingetreten ist. Die derzeitige Umweltpolitik wird tatsächlich genau in diesem Geist gestaltet: technokratisch, marktförmig, vorwiegend auf Großtechnologien setzend. Nichtkapitalistische Alternativen dazu werden mit der Autorität der fortgeschrittensten (Natur-)Wissenschaft häufig vom Tisch gefegt - symbolisch wie materiell. Diese Post-Politik verdankt ihre Rechtfertigung offenbar einer bestimmten Sichtweise auf den ökologischen Ausnahmezustand.

Christian Siefkes schließt den Bogen, den Ingo Schmidt eröffnet hat - wenn auch aus einer anderen Perspektive: Er fragt, welche Möglichkeiten der Befreiung von Arbeit die langfristigen Entwicklungstendenzen des Kapitalismus mit sich bringen, wenn nicht davon auszugehen ist, dass dieser - wie es prominent Paul Mason mit seiner These des „Postkapitalismus“vertritt - seine eigenen Reproduktionsbedingungen untergräbt.

In der Fortsetzung seines Beitrags zum Begriff der "Mitte“ in historischen und gegenwärtigen Klassenstudien diskutiert Ulf Kadritzke die aktuellen Mitte-Studien im Licht der klassentheoretischen Analysen der Weimarer Republik, die er in PROKLA 184 in Erinnerung rief und würdigte. Der zweite Teil verdeutlicht die politischen Konsequenzen des Begriffs der „Mitte“: „Wenig zu fürchten hat die Elite samt ihrem Beratungspersonal, solange es ihr gelingt, der angestellten Krankenphlegerin wie dem tariflosen Cloudworker ein Mitte-Dabeisein vorzuspiegeln und die Lehrerfamilie mit Eigenheim für das Steuervermeidungsinteresse der Plutokraten einzuspannen. Aufdie Selbsttäuschung dieser Mitte kann die Bourgeoisie der Gegenwart zumindest so lange zählen, wie ihr auf dem Feld der Begriffspolitik die Betreiber der Ungleichheitsforschung und der Themensalons zur Hand gehen.“. Die Auseinandersetzung mit Klassenverhältnissen liefert keine einfachen politischen Antworten - aber sie bildet die Voraussetzung für die Perspektive einer klassenlosen Gesellschaft, argumentiert Kadritzke.

Wenn die PROKLA aus der Druckerei kommt, ist das Jahr 2016 bald zu Ende und es beginnt die Zeit, in der viele gute und wichtige Initiativen um Spenden werben. Auch kritische Sozialwissenschaft und Zeitschriften wie die PROKLA sind nicht ohne solidarische Strukturen und finanzielle Unterstützung möglich. Seit 1971 erscheint die PROKLA und bietet politisch engagierte sozialwissenschaftliche und ökonomische Analysen, die gegenwärtig wichtiger sind denn je. Das zeigt ein Blick auf die letzten Ausgabenschwerpunkte. Der wissenschaftliche Zeitschriftenmarkt hat sich inzwischen selbst der Logik der neoliberalen Hochschule unterworfen. Für viele kritische Autoren und Autorinnen sind deshalb Zeitschriften wie die PROKLA unerlässlich, wenn sie Analysen jenseits des Mainstreams veröffentlichen und zur Diskussion stellen wollen. Texte online zu publizieren, hat sich bisher nicht als adäquate Alternative erwiesen, obwohl das Internet sicher an Bedeutung gewonnen hat und zukünftig noch wichtiger werden wird. Auch die PROKLA stellt ihre Ausgaben in einem Archiv als Volltext unter prokla.de zur Verfügung.

Allein von den Verkaufserlösen kann sich die PROKLA jedoch nicht finanzieren und in die Abhängigkeit von Parteien oder großen Verlagen wollte sie sich nie begeben. Deshalb wird die PROKLA von einem 
Förderverein herausgegeben, der „Vereinigung zur Kritik der politischen Ökonomie e.V.“, die jährlich in ihrer Vollversammlung die Redaktion der Zeitschrift wählt und die nächsten Themenschwerpunkte diskutiert.

Wir würden uns freuen, wenn die Leserschaft der PROKLA unsere Arbeit unterstützt. Vor allem regelmäßige finanzielle Beiträge der Vereinsmitglieder - und seien sie noch so klein - schaffen die Voraussetzungen für Kontinuität und Planbarkeit, wie sie für die Redaktionsarbeit unabdingbar sind. Für Mitgliedsbeiträge und Spenden stellen wir steuerabzugsfähige Spendenbescheinigungen aus, die „Vereinigung“ ist als gemeinnütziger Verein anerkannt. Weitere Informationen teilen wir gerne per E-Mail mit.

Ab 2017 müssen wir nach langer Zeit die Preise für die PROKLA geringfügig anheben. Der Abopreis beträgt ab dem 1.1.2017 42, $00 €$ (zzgl. Porto, statt vorher $38,00 €)$, der Preis für das Einzelheft ab der Ausgabe $18615,00 €$ (statt vorher $14,00 €$ ). Ältere Hefte können selbstverständlich zum alten Preis erworben werden; für AbonnentInnen der PROKLA gilt auch beim Bezug von Einzelheften der jeweilige Abopreis.

\section{Der PROKLA Förderverein}

Die PROKLA erscheint seit 1971 und bietet politisch engagierte sozialwissenschaftliche und ökonomische Analysen. Allein von den Verkaufserlösen kann sich die PROKLA nicht finanzieren und in die Abhängigkeit von Parteien oder großen Verlagen wollte sie sich nie begeben. Deshalb wird die PROKLA von einem Förderverein herausgegeben, der „Vereinigung zur Kritik der politischen Ökonomie e.V.“, die jährlich in ihrer Vollversammlung die Redaktion der Zeitschrift wählt und die nächsten Themenschwerpunkte diskutiert.

Kritische Sozialwissenschaft kann nicht dem Markt überlassen werden. Ohne solidarische Strukturen und finanzielle Unterstützung sind Zeitschriften wie die PROKLA kaum möglich. Die regelmäßigen finanziellen Beiträge der Vereinsmitglieder ermöglichen das Erscheinen der PROKLA, sie schaffen die Voraussetzungen für Kontinuität und Planbarkeit, wie sie für die Redaktionsarbeit unabdingbar sind. Wir freuen uns über weitere Mitglieder, regelmäßige Spenden oder einmalige Zuwendungen. Weitere Informationen teilen wir gerne perE-Mailmit (redaktion@prokla.de).

Vereinigung zur Kritik der politischen Ökonomie e.V.

IBAN: DE17 100100100538135100 - BIC: PBNKDEFF

Postbank Berlin 


\section{PROKLA 186 Die Materialität und politische Ökonomie des Internets (März 2017)}

Das Internet hatte lange Zeit den Ruf, ein dezentraler, hierarchiefreier und demokratischer Raum zu sein. Spätestens mit den Snowden-Enthüllungen wurde jedoch deutlich, dass die materielle Infrastruktur des Internets alles andere als das ist. Es gibt riesige und nur wenige Internet-Knotenpunkte, die die gesamten transatlantischen Datenströme konzentrieren. Zudem ist die Ökonomie des Internets sehr stark von Oligopolen geprägt. Das Internet ist zudem wie der geographische Raum von topographischen Verbindungen, Grenzen und Zentren und einer Peripherie geprägt - und ist vor allem ein „westliches“ Phänomen. Große Teile der Welt sind kaum „vernetzt“. Während in letzter Zeit viel über Datensicherheit und -schutz sowie über Kontrolle von geheimdienstlichen Aktivitäten diskutiert wurde, bleibt die "politische Ökonomie“ und die „Materialität" des Internets meist außerhalb kritischer Analyse. Es wird zwar viel darüber geredet, wie das Internet die Arbeitswelt verändern könnte („Industrie 4.0“), aber weniger darüber, wie das, was das Internet materiell und „immateriell“ ausmacht, selbst eine Anlagesphäre von Kapital sowie ein politisch und ökonomisch umkämpftes Terrain ist.

\section{PROKLA 187 Arbeit und Wertschöpfung im digitalen Kapitalismus (Juni 2017)}

Schlagwörter wie „Industrie 4.0“ oder „Digitalisierung“ sind derzeit in aller Munde. Doch die Verkündigung einer vierten industriellen Revolution wirkt angesichts der wirtschaftlichen Stagnation in Europa wie ein zweckoptimistisches Heilsversprechen. Der Begriff ist vielmehr ein rhetorischer Coup von Unternehmensverbänden und Bundesregierung: Der Standort Deutschland soll, so die Protagonisten, neu erfunden werden. Der aufgeladene Kunstbegriff „Industrie 4.0“ ist deshalb ungeeignet, einen realistischen Blick auf die aktuellen Veränderungen zu gewinnen. PROKLA 187 soll einen Beitrag zu einer kritischen Bestandsaufnahme der gegenwärtigen Umstrukturierungen leisten. Der Schwerpunkt soll hierbei auf neue Organisations- und Steuerungsformen von Wertschöpfung liegen, die Ansätze für eine Transformation des Kapitalismus enthalten und nachhaltige Veränderungen in der Nutzung von Arbeitskraft mit sich bringen - und was das für gesellschaftliche und gewerkschaftliche Kämpfe bedeutet. Jenseits der technologiezentrierten Engführungen, die den Diskurs um „Industrie 4.0“ prägen, wird eine herrschaftskritische Perspektive auf die gegenwärtige Rationalisierungsdynamik entwickelt. 